Find Your Balance at the

ASTS $19^{\text {th }}$ Annual State of the Art Winter Symposium

January $10-13,2019$

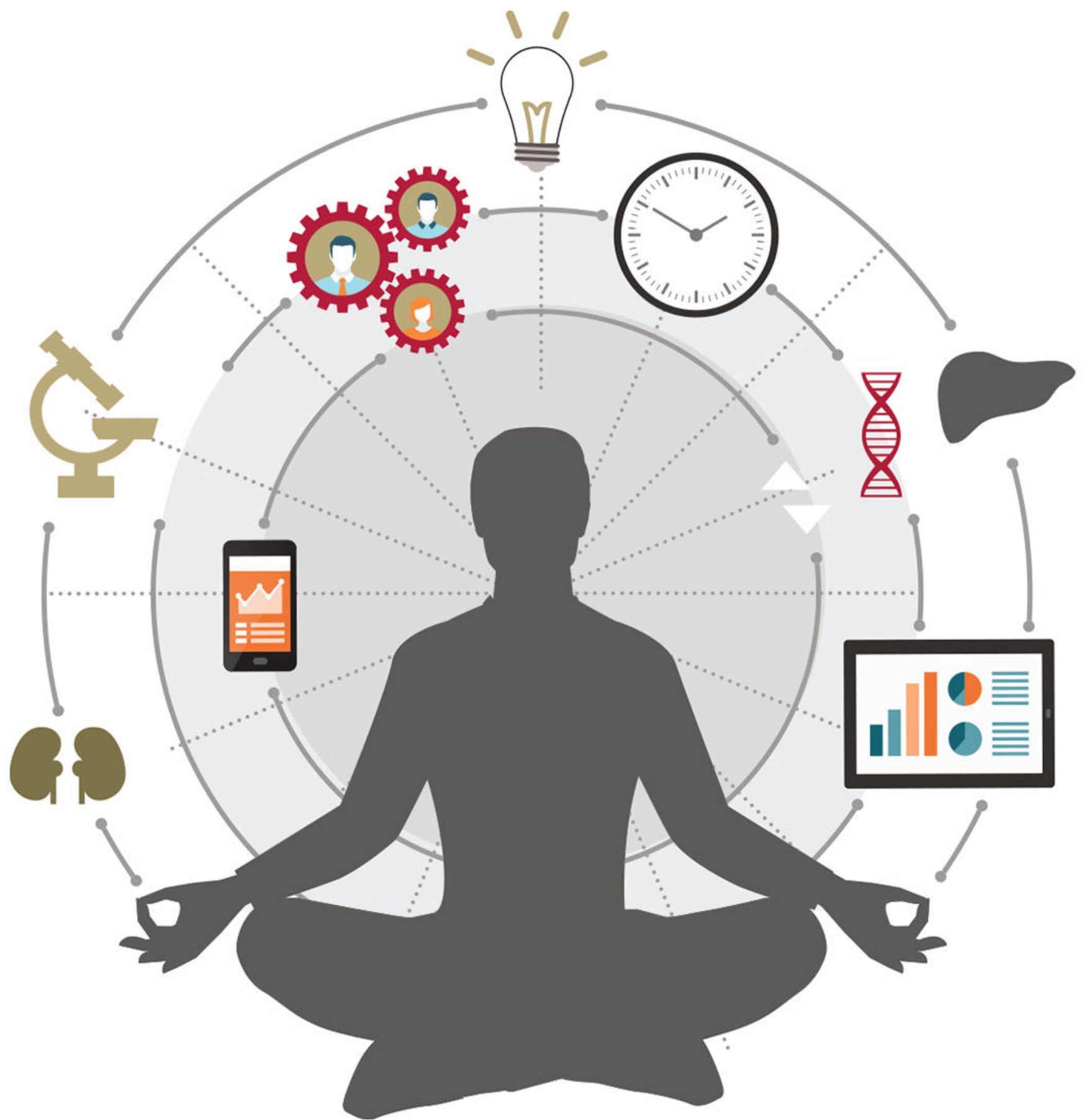

The Modern Transplant Surgeon: Striking the Right Balance

Register today at 


\title{
Impact of hepatic encephalopathy on liver transplant waiting list mortality in regions with different transplantation rates
}

\author{
Annarein J. C. Kerbert ${ }^{1}$ (D) | Enric Reverter ${ }^{2}$ | Lara Verbruggen $^{1} \mid$ Madelon Tieleman $^{3}$ | \\ Miguel Navasa ${ }^{2}$ | Bart J. A. Mertens ${ }^{4}$ | Sergio Rodríguez-Tajes ${ }^{2}$ | Marleen de Vree ${ }^{5}$ | \\ Herold J. Metselaar ${ }^{3}$ | Fang W. T. Chiang ${ }^{1}$ | Hein W. Verspaget ${ }^{1}$ | Bart van Hoek ${ }^{1}$ (D) | \\ Jaime Bosch $^{2,6}$ | Minneke J. Coenraad ${ }^{1}$
}

${ }^{1}$ Department of Gastroenterology and Hepatology, Leiden University Medical Center, Leiden, The Netherlands

${ }^{2}$ Liver Unit, Hospital Clínic, IDIBAPS, CIBEREHD, University of Barcelona,

Barcelona, Spain

${ }^{3}$ Department of Gastroenterology and Hepatology, Erasmus Medical Center, Rotterdam, The Netherlands

${ }^{4}$ Department of Medical Statistics and Bio-Informatics, Leiden University Medical Center, Leiden, The Netherlands

${ }^{5}$ Department of Gastroenterology and Hepatology, University Medical Center Groningen, Groningen, The Netherlands

${ }^{6}$ Swiss Liver Centre, Inselspital, Bern University, Bern, Switzerland

Correspondence

Annarein J. C. Kerbert, Leiden University Medical Center, Leiden, The Netherlands. Email: j.c.kerbert@lumc.nl

Funding information

This research was financially supported by a research grant from the Leiden University Medical Center (number: 8219-70550).

\begin{abstract}
Overt hepatic encephalopathy (OHE) negatively impacts the prognosis of liver transplant candidates. However, it is not taken into account in most prioritizing organ allocation systems. We aimed to assess the impact of OHE on waitlist mortality in 3 cohorts of cirrhotic patients awaiting liver transplantation, with differences in the composition of patient population, transplantation policy, and transplantation rates. These cohorts were derived from two centers in the Netherlands (reference and validation cohort, $\mathrm{n}=246$ and $\mathrm{n}=205$, respectively) and one in Spain (validation cohort, $n=253$ ). Competing-risk regression analysis was applied to assess the association of OHE with 1-year waitlist mortality. OHE was found to be associated with mortality, independently of MELD score, other cirrhosis-related complications and hepatocellular carcinoma (HCC; $\mathrm{sHR}=4.19,95 \% \mathrm{Cl}=1.9-9.5, P=0.001)$. The addition of extra MELD points for OHE counteracted its negative impact on survival. These findings were confirmed in the Dutch validation cohort, whereas in the Spanish cohort, containing a significantly greater proportion of HCC and with higher transplantation rates, OHE was not associated with mortality. In conclusion, OHE is an independent risk factor for 1-year waitlist mortality and might be a prioritization rule for organ allocation. However, its impact seems to be attenuated in settings with significantly higher transplantation rates.
\end{abstract}

\section{KEYWORDS}

hepatic encephalopathy, liver disease, organ allocation

\section{1 | INTRODUCTION}

Liver transplantation (LT) is a curative treatment option for patients with end-stage chronic liver disease and has a 5-year survival rate of 70\%-80\%. ${ }^{1}$ Prioritization of patients for LT is currently determined by the Model for End-Stage Liver Disease (MELD) score in most Western countries. This score, initially designed for assessing prognosis in patients undergoing transjugular intrahepatic portosystemic shunt (TIPS), incorporates objective markers of liver function (bilirubin, creatinine and the international normalized ratio, INR). ${ }^{2,3}$ The MELD score has been proven to be a strong prognostic indicator in many settings of cirrhosis, including organ allocation in patients registered on the transplant waiting list. However, it does not weight the effects of decompensation of liver disease such as ascites or hepatic encephalopathy (HE). HE is a neuropsychiatric syndrome caused by liver dysfunction in acute or chronic liver disease and/or portosystemic shunting. It describes a broad spectrum of neuropsychiatric abnormalities ranging from subclinical alterations to coma. ${ }^{4}$ It is associated 
with a substantial burden on caregivers and the overall healthcare system and severely impacts on the patients' health-related quality of life. ${ }^{5,6}$ Recent data show that HE remains a leading cause for readmission and mortality in patients with chronic liver disease. ${ }^{7-11}$ However, the subjectivity and inter-observer variability in diagnosing and grading of $\mathrm{HE}$ hampered the incorporation of $\mathrm{HE}$ in current liver allocation strategies. Nevertheless, several previous studies reported data confirming the prognostic relevance of the presence and severity of $\mathrm{HE}$, regardless of the MELD score. The main findings in this context so far are: (a) MELD score underestimates the risk of mortality in cirrhotic patients with $\mathrm{HE}^{12-15}$; (b) the severity of $\mathrm{HE}$ appears not to be correlated with the MELD score ${ }^{10,12}$; (c) the presence of high $\mathrm{HE}$ grades at time of registration at the waiting list increases 90-day waitlist mortality, independently of MELD score ${ }^{16}$ and (d) incorporation of HE in the MELD score may improve its prognostic ability. ${ }^{15}$

We aimed at assessing the impact of overt (clinically apparent) $\mathrm{HE}(\mathrm{OHE})$ on waitlist mortality in two independent, Western European cohorts (the Netherlands) of cirrhotic patients awaiting LT. In addition, we aimed at validating the impact of OHE on waitlist mortality in a Southern European cohort (Spain) with fundamental differences in the composition of patient population, transplantation policy and expected waiting time.

\section{2 | PATIENTS AND METHODS}

\section{1 | Study design}

Cirrhotic patients (age $>18$ years), who were registered at the waiting list for LT between 2007 and 2012, were retrospectively enrolled in the study. The study protocol conformed to the ethical guidelines of the 1975 Declaration of Helsinki as reflected a priori by the individual institution's Medical Ethics Committees. Exclusion criteria were enlistment for re-transplantation, combined liver and kidney transplantation, acute liver failure, patients with previous TIPS placement, hepatocellular carcinoma ( $\mathrm{HCC}$ ) outside Milan criteria and other advanced extrahepatic diseases. Demographics, clinical characteristics and laboratory values at the time of registration at the waiting list for LT were retrieved from patient files. A window of minus 2 weeks was applied for the presence of complications of cirrhosis at enlistment (ie, ascites, spontaneous bacterial peritonitis (SBP), variceal bleeding). Ascites was defined as either the development of de novo ascites requiring initiation of diuretics or worsening of previous existent ascites requiring an increase in diuretic treatment dose or large-volume paracentesis. Grading of HE severity as reported in the patient files was based on the West Haven Criteria (WHC), which is considered the gold standard for diagnosing and grading of $\mathrm{HE} .{ }^{17}$ Based on clinical criteria, this tool categorizes $\mathrm{HE}$ in five stages (ie, minimal HE and grade I-IV HE). Whereas it reliably distinguishes between patients with low vs high-grade HE, it has its weakness in discriminating between patients with grade I HE and those with no $\mathrm{HE}$ or minimal $\mathrm{HE}(\mathrm{mHE}) .{ }^{10,17}$ For this study, we, therefore, assessed the prognostic impact of an episode of OHE (ie, WHC grade II-IV ${ }^{4}$ ) as reported in the patients' medical records. Patients with any present (ie, at time of registration at the waiting list) or previous episode of $\mathrm{OHE}$ (pOHE) reported by the physician at or during a hospitalization were considered as "HE patients". All patients were followed up until death or LT with a maximum follow-up period of 12 months.

\subsection{Study cohorts}

The study consisted of 3 independent cohorts of cirrhotic patients who were registered at the waiting list for LT between 2007 and 2012: 1 reference cohort from 2 Dutch tertiary referral centers $(n=246)$, a validation cohort from another tertiary referral center in the Netherlands $(n=205)$ and a validation cohort from Spain $(n=253)$. Primary indications for enlistment were advanced liver cirrhosis or HCC in the setting of liver cirrhosis.

\section{3 | Statistical analysis}

Comparisons between patients with and without pOHE at time of registration at the waiting list were performed using the chi-square test or Student's $t$ test when appropriate. Baseline characteristics of patients in the three different cohorts were compared using the chisquare or ANOVA test when appropriate. Results are presented as frequencies and percentages or mean and standard deviation (SD) or median and interquartile range (IQR), when appropriate. $A P \leq 0.05$ was considered statistically significant. A competing-risk regression analysis using the method of Fine and $\mathrm{Gray}^{18}$ was performed in order to identify independent prognostic factors for mortality. Competingrisk analysis provides event-specific sub-distribution hazard ratios (sHR) that are adjusted for the interdependence of the impact of LT on mortality and vice versa. Mortality at the waiting list was the outcome of interest and LT was considered as a competing risk. Variables with a $P<0.10$ in univariate analysis, were included in multivariate analysis. Since laboratory MELD score is usually not reflective for the risk of mortality in patients with HCC, we performed multivariate models both with and without the inclusion of HCC patients. When analyzing global cohorts, HCC was also included in the multivariate models since it is a "favorable" factor for the transplantation rate, as extra MELD points have been added along the time of registration at the waiting list. With this approach, the relatively low MELD values for cirrhotic HCC patients with relatively preserved liver function were adjusted by including the variable HCC in the model.

After identifying the prognostic indicators for mortality at the waiting list, a "MELD-HE score" was calculated in both Dutch cohorts as previously proposed by Lucidi et $\mathrm{al}^{15}$ by adding 7 extra points to the MELD score of pOHE patients. In their study cohort, this specific number of extra points for the presence of pOHE was found to optimally counteract misclassification by the MELD score in patients with pOHE and at a higher risk of death. We included this MELD-HE score in the multivariate survival model to assess any residual impact of $\mathrm{pOHE}$.

To further assess the optimal number of points to be added for the presence of $\mathrm{pOHE}$, we studied the prognostic performance 
of the aforementioned survival models by excluding the variable pOHE and adding a range of 3-10 extra points to the MELD score in pOHE patients. Performance of these models was assessed by the following measures: Harrell C-index, which is a natural extension of the area under the Receiver Operating Characteristic (ROC) curve in the context of right censored data in survival analysis; Somers' D, which is a measure of agreement between pairs of possibly dependent ordinal variables, ranging from -1 (no agreement, model with poor predictive ability) to +1 (total agreement, model with good predictive ability); and the Akaike Information Criterion (AIC), which estimates the relative quality of statistical models for a given data set. AIC estimates the quality of each model relative to the other models. The model with the lowest AIC value is considered the preferred model. We identified the model with the number of extra MELD points for $\mathrm{POHE}$ that showed the best values for these three performance measures as compared to the reference model including the laboratory MELD score and the variable pOHE.
Statistical analysis was performed using the SPSS statistical package 20.0 (SPSS Inc, Chicago, IL, USA). Competing-risk survival analysis and prognostic measures were performed with the extension commands UAB Competing Risks and UAB AllSetsReg developed by the Applied Biostatistics Laboratory (Autonomous University of Barcelona).

\section{3 | RESULTS}

\section{1 | Reference cohort}

\subsection{1 | Patient characteristics}

Patient demographics and clinical characteristics at the time of registration at the waiting list for LT are shown in Table 1. A total of 83 patients had documented $\mathrm{HE}$ at time of registration at the LT waiting list (grade I: $n=36$, grade II: $n=31$, grade III: $n=14$, grade IV: $n=2$ ). Therefore, the study population contained 47 patients with pOHE (ie,
TABLE 1 Patient characteristics at time of registration at the waiting list for liver transplantation in the reference cohort $(n=246)$

\begin{tabular}{|c|c|c|c|c|}
\hline Variable & $\begin{array}{l}\text { All patients } \\
(n=246)\end{array}$ & $\begin{array}{l}\text { pOHE } \\
(n=47)\end{array}$ & $\begin{array}{l}\text { No pOHE } \\
(n=199)\end{array}$ & $P$-value \\
\hline Age (y), mean (SD) & $53.2(11.5)$ & $56.2(8)$ & $52.7(12)$ & 0.059 \\
\hline Gender (male), n (\%) & $174(70.7)$ & $31(66)$ & $143(71.9)$ & 0.477 \\
\hline \multicolumn{5}{|l|}{ Etiology, n (\%) } \\
\hline Alcohol & $73(29.7)$ & $22(46.8)$ & $51(25.6)$ & \multirow[t]{5}{*}{0.017} \\
\hline Viral hepatitis & $45(18.3)$ & $4(8.5)$ & $41(20.6)$ & \\
\hline $\mathrm{PSC} / \mathrm{PBC} / \mathrm{AlH}$ & $75(30.5)$ & $11(23.4)$ & $64(32.2)$ & \\
\hline NASH & $16(6.5)$ & 5 (10.6) & $11(5.5)$ & \\
\hline Other & $37(15)$ & $5(10.6)$ & $32(16.1)$ & \\
\hline \multicolumn{5}{|l|}{ Clinical features, n (\%) } \\
\hline $\mathrm{HCC}$ & $59(24)$ & $6(12.8)$ & $53(26.6)$ & 0.045 \\
\hline Ascites & $102(41.5)$ & $28(59.6)$ & $74(37.2)$ & 0.005 \\
\hline Variceal bleeding & $11(4.5)$ & 5 (10.6) & $6(3)$ & 0.023 \\
\hline SBP & $12(4.9)$ & $5(10.6)$ & $7(3.5)$ & 0.042 \\
\hline HRS & $10(4.1)$ & $7(14.9)$ & $3(1.5)$ & $<0.001$ \\
\hline \multicolumn{5}{|c|}{ Prognostic scores, median (IQR) } \\
\hline MELD & $12.2(9.5-16.3)$ & $15.4(11.8-18.9)$ & $11.3(8.9-15.2)$ & $<0.001$ \\
\hline Child-Pugh & $8(6-10)$ & $10(8-12)$ & $7(5-9)$ & $<0.001$ \\
\hline \multicolumn{5}{|c|}{ Laboratory data, mean (SD) } \\
\hline Creatinine $(\mu \mathrm{mol} / \mathrm{L})$ & $83.4(57.9)$ & $104.3(108.5)$ & $78.4(35.8)$ & 0.004 \\
\hline Sodium (mmol/L) & $138(4.6)$ & $137.7(5.3)$ & $138(4.5)$ & 0.43 \\
\hline INR & $1.29(0.35)$ & $1.46(0.47)$ & $1.25(0.3)$ & $<0.001$ \\
\hline Bilirubin $(\mu \mathrm{mol} / \mathrm{L})$ & 76.7 (128.9) & $147.1(215.6)$ & $60.1(91.1)$ & $<0.001$ \\
\hline Albumin $(\mathrm{g} / \mathrm{L})$ & $34.7(6.5)$ & $32.2(6.0)$ & $35.3(6.4)$ & 0.003 \\
\hline $\mathrm{AF}(\mathrm{U} / \mathrm{L})$ & $184(137.2)$ & $134(73)$ & $196(146)$ & $<0.001$ \\
\hline Leucocytes (x 109/L) & $5.8(2.9)$ & $6.69(3.9)$ & $5.60(2.6)$ & 0.036 \\
\hline
\end{tabular}

$\mathrm{AF}$, alkaline phosphatase; AIH, autoimmune hepatitis; HCC, hepatocellular carcinoma; HRS, hepatorenal syndrome; INR, international normalized ratio; IQR, interquartile range; MELD, model of endstage liver disease; PBC, primary biliary cirrhosis; $\mathrm{POHE}$, previous or present hepatic encephalopathy at time of registration at the waiting list; PSC, primary sclerosing cholangitis; SBP, spontaneous bacterial peritonitis; SD, standard deviation. 
zgrade $\mathrm{II} \mathrm{HE)}$. Of them, 26 had present $\mathrm{OHE}, 21$ had previous $\mathrm{OHE}$, and 7 had both present and previous OHE. Among patients without pOHE ( $n=199$ ), 7 (3.5\%) developed an episode of OHE during the 12-month follow-up period. As expected, patients with pOHE had more severe liver disease as compared to patients without $\mathrm{POHE}$ as reflected by higher MELD and CP scores, more elevated liver and kidney function parameters and higher prevalence of ascites and HRS.

\subsection{2 | Competing-risk survival analysis}

At 12 months of follow-up, 24 (9.8\%) patients had died while awaiting LT. Patients without pOHE at time of registration at the LT waiting list showed significantly better 1-year survival rates. One-year cumulative mortality was $25.5 \%(12 / 47)$ in pOHE patients vs $6.0 \%$ $(12 / 199)$ in non-pOHE patients $(P<0.001)$, resulting in a lower transplant rate in $\mathrm{pOHE}$ patients $(22 / 47,46.8 \%)$ as compared to nonpOHE patients (115/199, 57.8\%).

Previous or present hepatic encephalopathy at time of registration at the waiting list ( $\mathrm{OOHE}$ ) and other potential risk factors for 1-year waiting list mortality were analyzed in a univariate competing-risk regression model. Significant associations with mortality were found for MELD and CP score and the presence of pOHE, ascites, SBP and HRS at time of registration at the LT waiting list (Table 2). In multivariate analysis, pOHE was significantly associated with 1-year mortality at the LT waiting list in both the complete study cohort and in the sub-cohort excluding HCC patients, showing similar sub-Hazard ratios (Table 3A). Cumulative incidence function curves for mortality at the waiting list stratified by $\mathrm{POHE}$ are shown in Figure 1.

\section{2 | Validation cohorts}

Baseline characteristics of the Dutch and Spanish validation cohort are shown in Table S1. Baseline characteristics of the two Dutch cohorts were highly comparable, whereas the Spanish cohort had some fundamental differences in baseline characteristics as compared to the reference cohort. In the Spanish cohort, patients were older (57.1 vs 53.2 and 52.7 years, $P<0.001$ ) and had a significantly higher prevalence of pOHE and HCC as compared to the reference cohort (pOHE: $29.6 \%$ vs $19.1 \%$ and $15.6 \%, P<0.001$ and HCC: $48.6 \%$ vs $24.0 \%$ and $29.3 \%, P=0.001)$. Patients in the Spanish cohort had a significantly shorter median time until OLT or death (5.1 vs 6.8 and 8.2 months, $P<0.001$ ). As compared to the Dutch cohorts, the Spanish cohort had a higher cumulative 1-year transplantation rate (74.7\% vs $55.7 \%$ and $48.3 \%, P<0.001$ ) and a lower 1 -year cumulative waitlist mortality (6.3\% vs $9.8 \%$ and $12.7 \%, P<0.001)$.

To validate the impact of pOHE on 1-year mortality at the LT waiting list, identical competing-risk regression models were fitted for the two validation cohorts. Results of univariate analyses are shown in Table 3B,C.

In the Dutch validation cohort, it was confirmed that $\mathrm{pOHE}$ is a predictor for mortality at the waiting list, independently of MELD score and presence of HCC and ascites (Table 3B). Cumulative incidence curves for mortality at the waiting list stratified for $\mathrm{POHE}$ in the Dutch validation cohort are shown in Figure 2. However, in contrast to the findings in the two Dutch cohorts, pOHE was not associated with 1-year mortality at the waitlist in the Spanish cohort. In this cohort, leucocytes and ascites were associated with mortality in multivariate analysis, although these results must

TAB LE 2 Results of univariate competing-risk regression analysis of potential risk factors for 1-year mortality in the Dutch reference cohort, the Dutch validation cohort, and the Spanish validation cohort

\begin{tabular}{|c|c|c|c|c|c|c|}
\hline Cohort & \multicolumn{2}{|c|}{ Reference $(n=246)$} & \multicolumn{2}{|c|}{ Dutch validation $(n=205)$} & \multicolumn{2}{|c|}{ Spanish validation $(n=253)$} \\
\hline Gender male & $1.25(0.53-2.93)$ & 0.606 & $1.08(0.47-2.49)$ & 0.858 & $3.23(1.22-8.57)$ & 0.018 \\
\hline Albumin & $0.96(0.9-1.02)$ & 0.167 & $0.91(0.85-0.96)$ & 0.002 & $0.95(0.89-1.00)$ & 0.068 \\
\hline Child-Pugh score & $1.26(1.05-1.53)$ & 0.015 & $1.64(1.35-2.0)$ & $<0.001$ & $1.12(0.93-1.34)$ & 0.229 \\
\hline MELD score & $1.08(1.01-1.15)$ & 0.017 & $1.16(1.08-1.24)$ & $<0.001$ & $1.06(0.96-1.17)$ & 0.219 \\
\hline pOHE & $4.89(2.2-10.83)$ & $<0.001$ & $3.31(1.47-7.47)$ & 0.004 & $0.53(0.15-1.85)$ & 0.534 \\
\hline $\mathrm{HCC}$ & $1.90(0.84-4.27)$ & 0.123 & $0.191(0.04-0.82)$ & 0.026 & $0.82(0.31-2.19)$ & 0.693 \\
\hline Variceal bleeding ${ }^{a}$ & $3.55(1.02-12.3)$ & 0.046 & $1.51(0.78-2.32)$ & 0.254 & - & - \\
\hline
\end{tabular}

HCC, hepatocellular carcinoma; MELD, model of end-stage liver disease; pOHE, present or previous hepatic encephalopathy at time of registration at the waiting list; SBP, spontaneous bacterial peritonitis; sHR, sub-distribution hazard ratio for mortality at the waiting list; $95 \% \mathrm{Cl}$, $95 \%$ confidence interval.

${ }^{a}$ Competing-risk regression analysis could not be performed for these variables because of few events in these subgroups. 
TAB LE 3 Multivariate competing-risk survival analysis assessing 1-year waitlist mortality with and without inclusion of HCC patients in (A) the reference cohort, (B) the Dutch validation cohort and (C) the Spanish validation cohort

\begin{tabular}{|c|c|c|c|c|}
\hline \multicolumn{5}{|c|}{ A. Reference cohort $(n=246)$} \\
\hline pOHE & $4.19(1.85-9.51)$ & 0.001 & $5.58(1.87-16.67)$ & 0.002 \\
\hline MELD score & $1.06(1.01-1.12)$ & 0.027 & $1.07(1.01-1.12)$ & 0.023 \\
\hline \multicolumn{5}{|c|}{ B. Dutch validation cohort $(n=205)$} \\
\hline \multirow[b]{2}{*}{ Variables } & \multicolumn{2}{|c|}{ All patients $(n=205)$} & \multicolumn{2}{|c|}{ Non-HCC patients $(n=145)$} \\
\hline & $\operatorname{sHR}(95 \% \mathrm{Cl})$ & $P$-value & $\operatorname{sHR}(95 \% \mathrm{Cl})$ & $P$-value \\
\hline $\mathrm{HCC}$ & $0.52(0.10-2.58)$ & 0.420 & N.A. & N.A. \\
\hline \multicolumn{5}{|c|}{ C. Spanish validation cohort $(n=253)$} \\
\hline \multirow[b]{2}{*}{ Variables } & \multicolumn{2}{|c|}{ All patients $(n=253)$} & \multicolumn{2}{|c|}{ Non-HCC patients $(n=130)$} \\
\hline & sHR $(95 \% \mathrm{Cl})$ & $P$-value & sHR $(95 \% \mathrm{CI})$ & $P$-value \\
\hline Ascites & $5.03(1.24-20.44)$ & 0.024 & $5.10(0.60-43.18)$ & 0.135 \\
\hline MELD score & $1.05(0.89-1.23)$ & 0.578 & $0.99(0.81-1.22)$ & 0.945 \\
\hline Leucocytes & $1.32(1.10-1.57)$ & 0.002 & $1.40(1.14-1.71)$ & 0.001 \\
\hline $\mathrm{HCC}$ & $3.49(0.78-15.59)$ & 0.102 & N.A. & N.A. \\
\hline
\end{tabular}

$\mathrm{Cl}$, confidence interval; HCC, hepatocellular carcinoma; MELD, model for end-stage liver disease; NA, not applicable; pOHE, previous or present hepatic encephalopathy at time of registration at the waiting list; SBP; spontaneous bacterial peritonitis; sHR, sub-distribution hazard ratio for 1-year mortality at the waiting list.

be interpreted cautiously due to the small number of events (16 deaths) (Table 3C).

\section{3 | Priority MELD score for pOHE patients?}

In the following analyses, we aimed at defining a prognostic model optimally adjusting for the negative impact of pOHE on 1-year waiting list mortality. Firstly, as previously proposed by Lucidi et al, ${ }^{15}$ seven extra points were added to the MELD score of pOHE patients (the "MELD-HE score") in the two Dutch cohorts. By including this MELD-HE score in the multivariate survival models, pOHE appeared not to be an independent prognostic factor for 1-year waiting list mortality, thereby confirming that extra MELD points may indeed counteract the negative impact of pOHE on survival (Table S2).

Secondly, we conducted multivariate competing-risk survival models including different MELD-HE scores with a range of three till 10 extra points for $\mathrm{POHE}$. With this approach, we aimed at assessing which number of extra points optimally counteracted the negative impact of pOHE on mortality and lead to the model with best predictive ability for 1-year survival as compared to the reference model including the laboratory MELD score and the variable pOHE (Table
S3). In consistence with Lucidi et al, we found that 7 extra MELD points for $\mathrm{pOHE}$ were needed to counteract the negative impact of pOHE on survival in our study population. The models with best predictive ability (as assessed by AIC, Harrell's C-index and Somers' D) were obtained with 7-9 extra points (Table S3A). For the Dutch validation cohort, the models that showed best predictive performance were those with 4-6 extra MELD points and 5 extra MELD points were needed to neutralize the impact of pOHE on survival (Table S3B). When combining the two Dutch cohorts, best predictive performance was found for the prognostic model including six extra MELD points for pOHE (Table S3C). Sub-analysis in non-HCC patients showed similar results (Table S3D).

\section{4 | DISCUSSION}

This study shows that the presence of $\mathrm{pOHE}$ at time of registration at the waiting list for $\mathrm{LT}$, is an independent risk factor for mortality awaiting LT in both cirrhotic patients with and without HCC. However, it has been found that pOHE seems not to be of prognostic significance in a cohort with significantly higher transplantation 

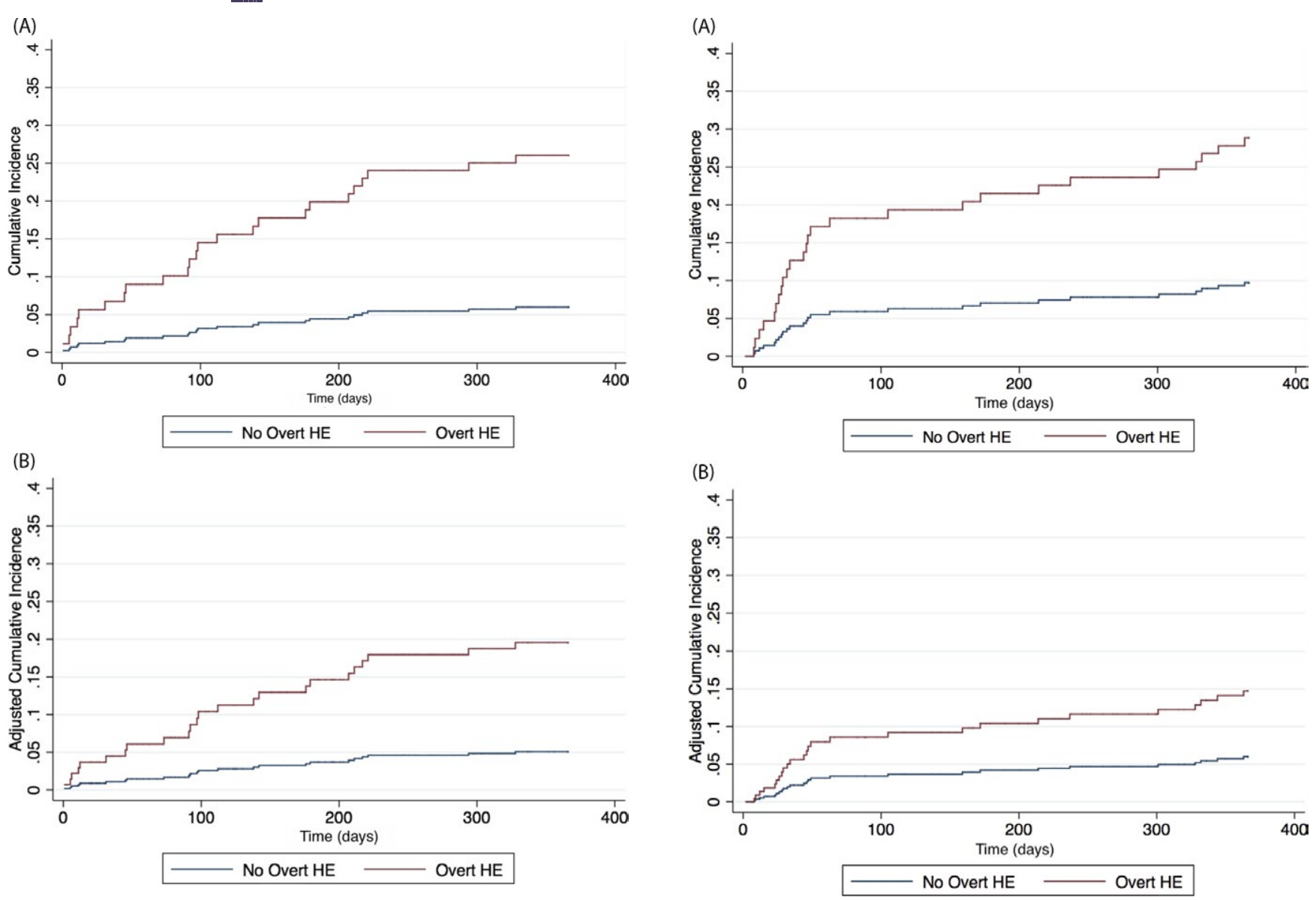

FIGURE 1 Cumulative incidence functions for mortality at the waiting list stratified by $\mathrm{POHE}$ in the Dutch reference cohort: (A) unadjusted effect of pOHE, (B) adjusted effect of pOHE by covariables (MELD, HCC, and baseline SBP). HCC, hepatocellular carcinoma; MELD, model of end-stage liver disease; pOHE, previous or present hepatic encephalopathy at time of registration at the waiting list; SBP, spontaneous bacterial peritonitis

rates and shorter waiting time until transplantation. Furthermore, it was validated in this study that the addition of extra MELD points for pOHE patients, as recently proposed by Lucidi et al, ${ }^{15}$ counteracts the negative impact of pOHE on waitlist mortality.

Several previous studies have reported a significant association for HE with an increased risk of waitlist mortality. ${ }^{12,13,16,19}$ Therefore, the hypothesis was raised that the lack of considering $\mathrm{HE}$ in the prioritizing criteria for LT may lead to underestimation of the severity of the underlying liver disease and prognosis. Indeed, subsequent studies have shown the additional prognostic value of considering HE in the MELD score. ${ }^{10,12,15,19}$ However, except for the recent study of Lucidi et al, ${ }^{15}$ these studies did not adjust for the presence of other complications of cirrhosis as risk factors for mortality at the liver transplant waiting list in patients with $\mathrm{HE}$. In addition, the majority of these studies did not perform competing-risk survival analysis, which is essential in the context of assessing waitlist mortality, since it provides event-specific hazard ratios without censoring of patients. Using this approach, our results confirm in two large cohorts of cirrhotic patients awaiting LT that HE is a risk factor for

FIGURE 2 Cumulative incidence functions for mortality at the waiting list stratified by overt $\mathrm{HE}$ in the Dutch validation cohort: (A) unadjusted effect of pOHE, (B) adjusted effect of pOHE by covariables (MELD, HCC, and baseline ascites). HCC, hepatocellular carcinoma; HE, hepatic encephalopathy; MELD, model of end-stage liver disease; pOHE, previous or present hepatic encephalopathy at time of registration at the waiting list

1-year mortality at the waiting list, independently of MELD score and other complications of cirrhosis. It must be noted that the analysis of the prognostic impact of $\mathrm{HE}$ was limited to patients with $\mathrm{OHE}$ (WHC II-IV), because of the more observator dependent, subjective assessment of minimal and grade I HE. Our finding that pOHE has a negative prognostic implication is, therefore, a robust finding, unlikely to be influenced by subjective judgment. The strategy to prioritize patients with pOHE with extra MELD points seems sensible and, as previously proposed by Lucidi et al, ${ }^{15}$ adding seven extra points to the laboratory MELD score optimally counteracted the negative effect of pOHE on survival in the study cohort. An immortal time bias could be suspected for these survival analyses as the (additional) prognostic impact of the development of OHE during follow-up was not assessed. However, the low number of patients who developed OHE during follow-up (3.5\%), makes this hypothesis unlikely.

The findings in the study cohort were confirmed in the Dutch validation cohort. In the Spanish cohort, however, pOHE was not a predictor for mortality at the waiting list. This may be explained 
by several relevant differences between the Dutch and Spanish cohorts. Firstly, the transplantation rate in the Spanish cohort was significantly higher and the waiting list time and mortality were lower than in the Dutch cohorts. Spain holds a leading position worldwide in deceased organ donation. Their unique organizational system resulted in $\mathbf{4 0}$ million organ donors per million population giving rise to the highest number of solid organ transplantations in the world in $2015 .{ }^{20}$ In this setting, complications of cirrhosis may have less impact on survival as compared to populations with a longer expected waiting list time. Accordingly, although the prevalence of pOHE was significantly higher in the Spanish cohort than in Dutch cohorts (29.6\% vs $16 \%$ and $19 \%$ ), it was not associated with mortality, which probably reinforces the role of a high transplantation rate to reduce the negative impact of pOHE. Secondly, prioritization rules for HCC differ between Spain and Netherlands: In Spain HCC patients enter the waiting list with 19 MELD points (and a subsequent increase by one point quarterly), while in the Netherlands an exception MELD score is granted from 6 months after enlistment. The above-described differences in organ allocation policy between countries may explain the shorter waiting times until LT in the Spanish cohort.

Some limitations according to the present study are to be considered. The most important one is the retrospective study design. The presence of $\mathrm{pOHE}$ relied on documentation in patient files. The subjectivity and inter-observer variability in diagnosing and grading of $\mathrm{HE}$ remains a challenging aspect in research to this neuropsychological syndrome. Prospective studies with clear definitions for diagnosing and grading of HE will be needed to validate its prognostic value on waitlist mortality and to evaluate its ability to prioritize patients for LT. Due to the retrospective study design, we were not able to reliably investigate the impact of the use of medication for $\mathrm{HE}$ and the use of other potentially relevant co-medication, such as betablockers and diuretics, on the survival of HE patients. It must also be noted that events occurring between registration at the waiting list and death or transplantation, such as variceal bleeding and SBP, were not recorded due to the cross-sectional design of the study. Of course, these intermediary events might account for mortality and could be better characterized and analyzed with a prospective design. Finally, in both Dutch cohorts, other complications of cirrhosis also appeared to have an independent impact on the waitlist mortality (SBP and ascites) suggesting that other factors may help in refining the priority rules for organ allocation. Finally, the low number of deaths in the Spanish cohort makes the statistical assessment in this cohort less robust than in the Dutch cohorts, thus making it more difficult to demonstrate a negative impact of $\mathrm{POHE}$ on waiting list mortality.

Based on the results of the present study, we conclude that the presence and/or a history of clinically symptomatic HE at time of registration at the waiting list for $L T$, is an independent risk factor for mortality and that it might be a prioritization rule for organ allocation. However, its prognostic impact seems to be attenuated in settings with significantly higher transplantation rates and shorter waiting time until transplantation.

\section{CONFLICT OF INTEREST}

None.

\section{AUTHORS' CONTRIBUTIONS}

Study concept and design: Minneke J. Coenraad, Jaime Bosch, Miguel Navasa; data collection: Annarein J. C. Kerbert, Enric Reverter, Lara Verbruggen, Madelon Tieleman, Fang W. T. Chiang; statistical analysis: Annarein J. C. Kerbert, Enric Reverter, Bart J. A. Mertens, Sergio Rodríguez-Tajes; analysis and interpretation of data: Annarein J. C. Kerbert, Enric Reverter, Minneke J. Coenraad, Jaime Bosch; drafting of the manuscript: Annarein J. C. Kerbert; critical revision of the manuscript: Enric Reverter, Lara Verbruggen, Madelon Tieleman, Miguel Navasa, Bart J. A. Mertens, Sergio Rodríguez-Tajes, Marleen de Vree, Herold J. Metselaar, Fang W. T. Chiang, Hein W. Verspaget, Bart van Hoek, Jaime Bosch, Minneke J. Coenraad.

\section{ORCID}

Annarein J. C. Kerbert (iD http://orcid.org/0000-0001-9845-2176

Bart van Hoek iD http://orcid.org/0000-0001-6527-764X

\section{REFERENCES}

1. Adam R, Karam V, Delvart V, et al. Evolution of indications and results of liver transplantation in Europe. A report from the European Transplant Registry (ELTR). J Hepatol. 2012;57:675-688.

2. Wiesner R, Edwards E, Freeman R, et al. Model for end-stage liver disease (MELD) and allocation of donor livers. Gastroenterology. 2003;124:91-96.

3. Montgomery A, Ferral H, Vasan R, Postoak DW. MELD score as a predictor of early death in patients undergoing elective transjugular intrahepatic postosystemic shunt (TIPS) procedures. Cardiovasc Intervent Radiol. 2005;28:307-312.

4. Vilstrup H, Amodio P, Bajaj J, et al. Hepatic encephalopathy in chronic liver disease: 2014 practice guideline by the American association for the study of liver diseases and the European association for the study of the liver. Hepatology. 2014;60:715-733.

5. Stepanova M, Mishra A, Venkatesan C, Younossi ZM. In-hospital mortality and economic burden associated with hepatic encephalopathy in the United States from 2005 to 2009. Clin Gastroenterol Hepatol. 2012;10:1034-1041.e1.

6. Poordad FF. Review article: the burden of hepatic encephalopathy. Aliment Pharmacol Ther. 2007;25:3-9.

7. D'Amico G, Morabito A, Pagliaro L, Marubini E. Survival and prognostic indicators in compensated and decompensated cirrhosis. Dig Dis Sci. 1986;31:468-475.

8. Amodio P, Del Piccolo F, Pettenò E, et al. Prevalence and prognostic value of quantified electroencephalogram (EEG) alterations in cirrhotic patients. J Hepatol. 2001;35:37-45.

9. Stewart CA, Maninchoc M, Kim WR, Kamath PS. Hepatic encephalopathy as a predictor of survival in patients with end-stage liver disease. Liver Transpl. 2007;13:1366-1371.

10. Bustamante J, Rimola A, Ventura PJ, et al. Prognostic significance of hepatic encephalopathy in patients with cirrhosis. J Hepatol. 1999;30:890-895.

11. Bajaj JS, Reddy KR, Tandon P, et al. The 3-month readmission rate remains unacceptably high in a large North American cohort of patients with cirrhosis. Hepatology. 2016;64:200-208. 
12. Yoo HY, Edwin D, Thuluvath PJ. Relationship of the model for endstage liver disease (MELD) scale to hepatic encephalopathy, as defined by electroencephalography and neuropsychometric testing, and ascites. Am J Gastroenterol. 2003;98:1395-1399.

13. Bajaj JS, Saeian K. MELD score does not discriminate against patients with hepatic encephalopathy. Dig Dis Sci. 2005;50:753-756.

14. Said A, Williams J, Holden J et al. Model for end-stage liver disease score predicts mortality across a broad spectrum of liver disease. J Hepatol. 2004;40:897-903.

15. Lucidi C, Corradini SG, Abraldes JG, et al. Hepatic encephalopathy expands the predictivity of model for end-stage liver disease in liver transplant setting: evidence by means of 2 independent cohorts. Liver Transpl. 2016;22:1333-1342.

16. Wong RJ, Gish RG, Ahmed A. Hepatic encephalopathy is associated with significantly increased mortality among patients awaiting liver transplantation. Liver Transpl. 2014;20:1454-1461.

17. Ferenci P, Lockwood A, Mullen K, Tarter R, Weissenborn K, Blei AT. Hepatic encephalopathy - definition, nomenclature, diagnosis, and quantification: final report of the working party at the 11th World Congresses of Gastroenterology, Vienna, 1998. Hepatology. 2002;35:716-721.

18. Fine JP, Gray RJ. A proportional hazards model for the subdistribution of a competing risk. J Amer Statist Assoc. 1999;94:496-509.
19. Ham J, Gish RG, Mullen K. Model for end-stage liver disease (MELD) exception for hepatic encephalopathy. Liver Transpl. 2006;12(suppl3):S102-S104.

20. Matesanz R, Domínguez-Gil, Coll E, et al. How Spain reached 40 deceased organ donors per million population. Am J Transplant. 2017;17:1447-1454.

\section{SUPPORTING INFORMATION}

Additional supporting information may be found online in the Supporting Information section at the end of the article.

How to cite this article: Kerbert AJC, Reverter E, Verbruggen L, et al. Impact of hepatic encephalopathy on liver transplant waiting list mortality in regions with different transplantation rates. Clin Transplant. 2018;32:e13412. https://doi. org/10.1111/ctr.13412 